\title{
PROGRAMA PARA EL DIMENSIONAMIENTO DE SOPORTES ESBELTOS DE HORMIGON ARMADO CON UN ORDENADOR DE BOLSILLO
}

\author{
(PROGRAM FOR THE DESIGN OF SLENDER REINFORCED CONCRETE COLUMNS ON A POCKET \\ COMPUTER)
}

Francisco Morán Cabré, Dr. Ingeniero de Caminos. IETCC/CSIC

Olga Río Suárez, Ingeniero de Caminos. IETcc/CSIC

\author{
RESUMEN \\ Se presenta un programa para el dimensionamiento de las \\ armaduras longitudinales de soportes esbeltos de hormigón \\ armado de sección rectangular sometidos a \\ flexocompresión recta. El programa calcula la armadura \\ estrictamente necesaria, dada la distribución de la misma \\ en la sección del soporte. El cálculo se realiza en teoría de \\ segundo orden por el Método de las Curvaturas de Referencia, \\ de acuerdo con la Instrucción Española y con el Código Modelo \\ del CEB. El programa está preparado para el microordenador \\ personal de bolsillo HP-75/71. Se incluye un listado en \\ lenguaje BASIC y ejemplos numéricos.
}

SUMMARY

A program for the design of the longitudinal reinforcement of slender reinforced concrete columns is presented. The column is supposed of rectangular cross-section and submitted to bending and compression $(M, N)$. The program obtains the minimum amount of longitudinal reinforcement, given the reinforcement arrangement. Second order theory is applied in accordance with Spanish Regulations and the CEB Model Code. The Reference Curvatures Method is adopted. The program has been written for the personal pocket microcomputer HP-75/71. The BASIC listing and numerical examples are included.

\section{Introducción. Objeto del programa}

A continuación se describe un programa para el dimensionamiento de la armadura longitudinal de soportes esbeltos de hormigón armado de sección rectangular sometidos a flexocompresión recta $(M, N)$.

El programa, llamado DMNP, está escrito en lenguaje BASIC para el ordenador de bolsillo HP-75/71. Ocupa unos $3 \mathrm{k}$ octetos de memoria y puede adaptarse con facilidad a cualquier otro ordenador personal. Forma parte de un conjunto de programas para el cálculo de estructuras de hormigón armado $\left(^{*}\right)$.

Las bases de cálculo son las contenidas en los artículos 36, «Principios generales de cálculo de secciones sometidas a solicitaciones normales», y 43, «Pandeo» de la Instrucción Española de Hormigón EH-82 (1), que coinciden con las del Código Modelo CM-78 del CEB. En lo que

$\left(^{*}\right)$ En publicaciones recientes se han descrito tres programas más del conjunto: el DMNE, para dimensionamiento de secciones rectangulares en flexocompresión recta (4); el $C M 2 R$, para la comprobación de secciones rectangulares en flexocompresión esviada (5), y el DM2G, para el dimensionamiento de secciones poligonales en flexocompresión esviada (6). respecta a las cuantías mínimas, se respeta la limitación de la Tabla 38.3 de la EH-82 para soportes armados con aceros de las calidades AEH-400, AEH-500 ó AEH-600, esto es, una cuantía geométrica mínima de la armadura longitudinal del cuatro por mil.

Se supone que tanto la sección como las armaduras longitudinales son constantes a lo largo del soporte. Se supone también que el soporte es aislado, biarticulado, y que el momento de primer orden $M$ es constante a lo largo del mismo. Para soportes que formen parte de pórticos o que no estén biarticulados será preciso obtener previamente la longitud de pandeo $I_{0}$, o longitud del soporte aislado biarticulado equivalente; para soportes con momentos de primer orden variables a lo largo de los mismos podrá adoptarse un momento de primer orden equivalente $M_{0}$. Para ello pueden aplicarse los apartados 43.1 .2 (comentario) y 43.5.2-b de la $\mathrm{EH}-82$, respectivamente.

El dimensionamiento se obtiene en forma de la cantidad de armadura estrictamente necesaria para resistir la solicitación actuante, eligiéndose como datos una determinada disposición de armado y un recubrimiento determinado. 


\section{Estructura y funcionamiento del programa. Datos y resultados}

La estruciura y el funcionamiento del programa son comunes a todos los programas del conjunto aludido en la introducción. En la preparación de dichos programas se han tenido en cuenta las características especiales de los microordenadores a los que van destinados (ordenadores de bolsillo de muy limitada capacidad de memoria y velocidad de cálculo), junto con los imperativos de comodidad de uso y de generalidad de aplicación. Estos condicionantes han conducido a la adopción de los siguientes criterios prioritarios en el diseño de los programas:

a) Tamaño mínimo de los programas, para su utilización en máquinas de memoria mínima.

b) Rapidez de cálculo máxima compatible con la precisión deseada en cada caso, para adaptarse a máquinas de limitada velocidad aritmética, lo que requiere el uso de algoritmos eficientes.

c) Entrada de datos cómoda e interactiva desde teclado, dando en cada caso el número de datos mínimo imprescindible para el problema concreto a resolver.

d) Posibilidad de efectuar recálculos con el mínimo esfuerzo, bien sea de una misma pieza o de distintas piezas en las que cambien sólo algunos datos, como sucede en la práctica del cálculo de estructuras de hormigón.

e) Planteamiento de la máxima generalidad, en relación con los problemas que se presentan más frecuentemente en la práctica del proyecto o de la comprobación de estructuras de hormigón.

f) Salida de resultados por pantalla, a ser posible de una sola línea, para prescindir de la necesidad del uso de una impresora, cuyo precio es del mismo orden de magnitud que el del microordenador.

Estos criterios han aconsejado preparar un conjunto de programas en lugar de uno solo que valga para todos los casos; de esta forma se consigue una mejor adaptación a las características concretas de cada problema. Por otra parte, estos criterios han conducido a una determinada estructura del programa, semejante a la de los otros programas del conjunto, que se describe a continuación.

El organigrama de DMNP puede verse en la figura 1. A continuación se describe su funcionamiento, detallando cuáles son y cómo

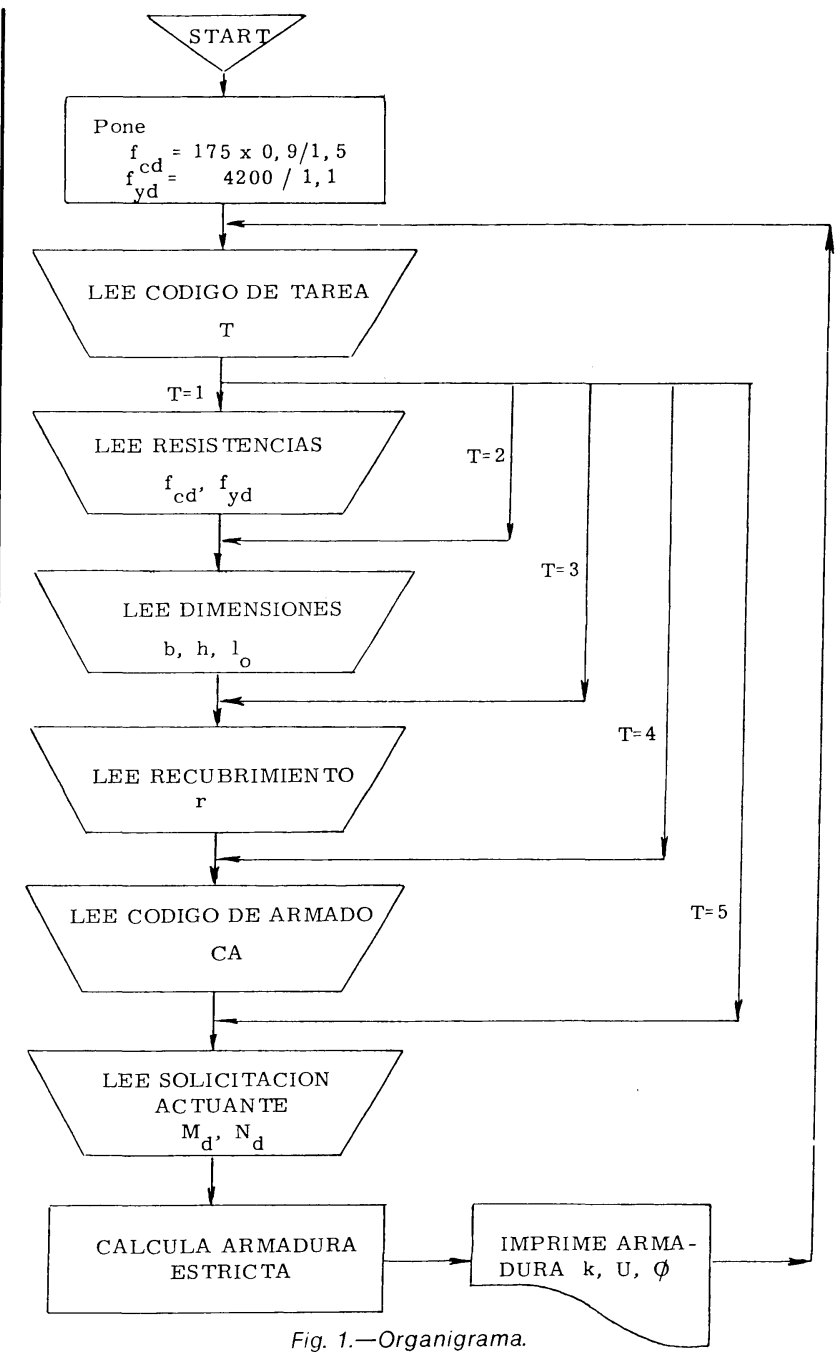

deben darse los datos, y cuáles son y cómo se imprimen los resultados.

Al comienzo, el programa pide y lee el código de tarea $\mathrm{T}$. Si se introduce $\mathrm{T}=1$ el programa pide y lee, a continuación, los valores de las resistencias de cálculo del hormigón y del acero $f_{c d} y f_{y d}$, en $\mathrm{kp} / \mathrm{cm}^{2}$. En caso de que se introduzca $\mathrm{T}=2$ el programa no lee estos datos,

manteniendo las resistencias usadas anteriormente. Si se arranca el programa con $\mathrm{T}=2$, se adoptan los siguientes valores, considerados como frecuentes en el momento de escribirse el programa:

$$
\begin{aligned}
\mathrm{f}_{\mathrm{cd}} & =175 \times 0,9 / 1,5=105 \mathrm{kp} / \mathrm{cm}^{2} ; \\
\mathrm{f}_{\mathrm{yd}} & =4.200 / 1,1=3.818 \mathrm{kp} / \mathrm{cm}^{2} .
\end{aligned}
$$

Naturalmente, si el usuario utiliza otros valores de las resistencias de cálculo con mayor frecuencia, bastará con que los introduzca, en el lugar adecuado del listado BASIC (ver apartado 6), sustituyendo a los anteriores. 


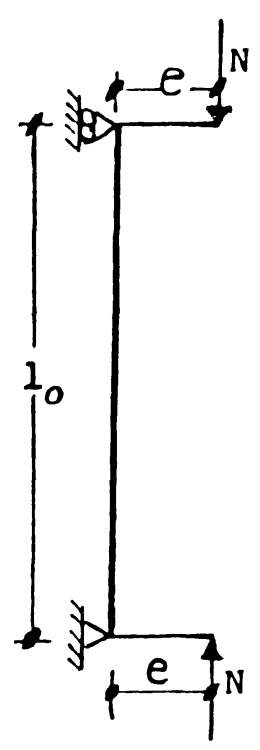

Fig. 2.-Definición del soporte.

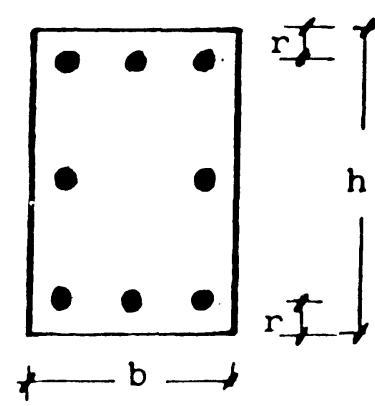

A continuación, o siempre que se haya introducido $\mathrm{T}=2$, el programa pide y lee las dimensiones de la sección $b$ y $h$ y la longitud de pandeo del soporte $I_{0}$ (ver figura 2 ), en $\mathrm{cm}$. Es importante tener en cuenta que la dimensión $h$, o canto del soporte, corresponde a la dirección de la flexión $M$ que solicita al mismo, y al plano en que se supone que se produce el pandeo. En soportes que pueden pandear en las dos direcciones y en los que $b<h$ puede ser necesario efectuar un dimensionamiento independiente en dirección $b$ tomando $M=0$. Para ello deberán cambiarse entre sí las dimensiones $b$ y $h$ (ver ejemplo en el apartado 7).

En cuanto a la longitud $I_{\alpha}$ es la longitud del soporte biarticulado equivalente, igual al producto de la longitud I del soporte por el factor de longitud de pandeo $\alpha$, que se obtiene teniendo en cuenta las condiciones de vinculación en los extremos del soporte si es aislado, y la relación de rigideces en dichos extremos si forma parte de un pórtico (apartado 43.1.2, comentario, de la EH-82).

A continuación, o siempre que se haya introducido $\mathrm{T}=3$, el programa pide y lee el recubrimiento mecánico $r$ en centímetros, distancia del centro de gravedad de la armadura al paramento de la sección. Como dicho recubrimiento, que es igual al recubrimiento libre $c$ más el diámetro del cerco $\varnothing_{t}$ más el semidiámetro de la barra longitudinal $\varnothing: \mathrm{r}=\mathrm{c}+\varnothing_{\mathrm{t}}+1 / 2 \varnothing$, no suele ser conocido a priori, es frecuente introducir un valor estimativo y luego corregirlo, en especial si al substituir el diámetro teórico de la barra longitudinal por el de la serie normalizada el margen de seguridad obtenido así lo aconseja.

A continuación, o siempre que se haya introducido $\mathrm{T}=4$, el programa pide y lee el código de armado CA, que indica cuál es la disposición de armado elegida por el proyectista entre las posibles, algunas de las cuales se indican, a título de ejemplo, en la figura 3. El primer dígito del código de armado indica el número de barras de las armaduras frontales, y debe ser un entero comprendido entre 1 y 9 , ambos inclusive. El segundo dígito indica el número de barras de las armaduras laterales o de capas de armaduras, y debe ser un entero comprendido entre 2 y 9 . Mediante el código de armado especial $C A=12$ se indica que las armaduras frontales tienen una sola barra. En este caso, el resultado será la cuantía mecánica $U$ en toneladas de dichas armaduras frontales, y el diámetro $\varnothing$ que tendrían en el caso hipotético de que se armara con una sola barra. Si CA $=12$ el valor de $\varnothing$ no.se utilizará, mientras que el valor de U servirá para entrar en una tabla de capacidades mecánicas correspondiente al acero utilizado y encontrar la combinación más conveniente $n, \varnothing$ de número de barras y diámetro de las mismas para las armaduras frontales.

A continuación, o siempre que se haya introducido $\mathrm{T}=5$, el programa pide y lee la solicitación de cálculo actuante $M_{d}, N_{d}$ en metros/toneladas y en toneladas, respectivamente. Como se ha indicado, estos valores deben estar mayorados, y el momento $M_{d}$ debe ser el de primer orden. Los esfuerzos $M_{d}$ y $N_{d}$ deben ser ambos positivos (si $M_{d}$ fuera negativo podría cambiársele de signo, teniendo en cuenta la simetría de la sección).

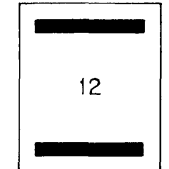

Fig. 3.-Ejemplos de Códigos de Armado CA.
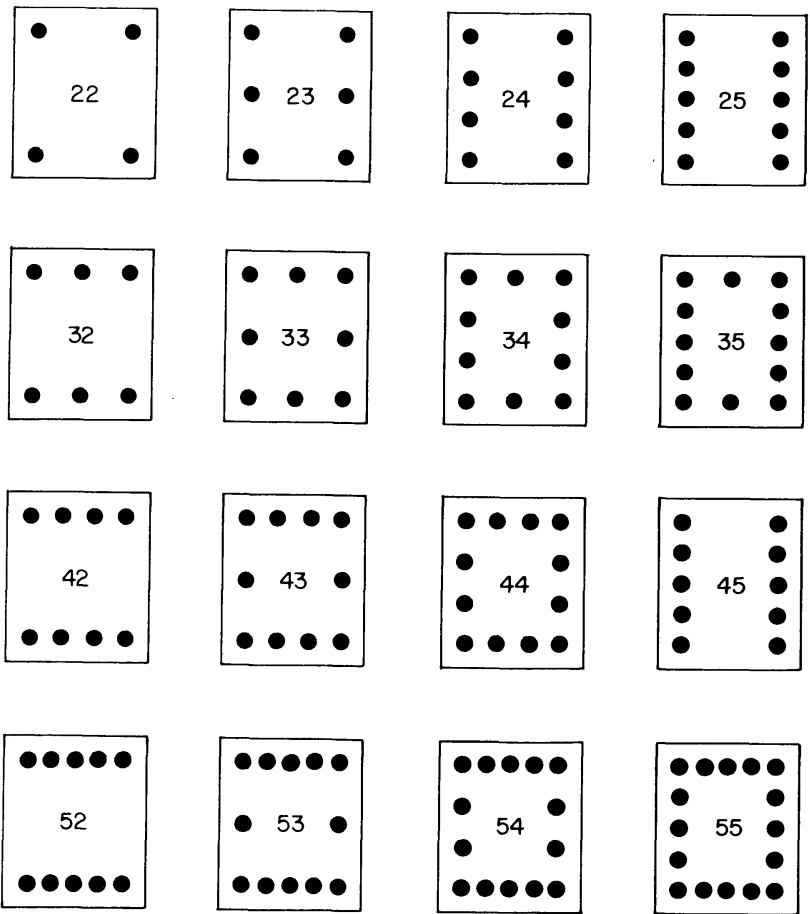
Con todos estos datos el programa está ya en condiciones de calcular las armaduras estrictamente necesarias para resistir la solicitación actuante, teniendo en cuenta los efectos de segundo orden, lo que se hace como se indica en el apartado siguiente. Al cabo de un tiempo de cálculo variable, y que puede estimarse en unos 25 segundos en el ordenador HP-75 y en unos 50 en el HP-71, aparecen en pantalla los valores de $k, \cup$ y $\varnothing$. El dígito $k$ indica cuál es el criterio que condiciona el

dimensionamiento del soporte, de acuerdo con el siguiente convenio:

$k=1$ si la armadura viene condicionada por el criterio de cuantía geométrica mínima del 4 por 1.000 (apartado 38.3 y tabla 38.3 de la Instrucción EH-82);

$k=2$ si la armadura es aquélla para la cual el soporte, bajo la solicitación de cálculo, alcanza un estado límite último de inestabilidad, es decir, un estado de equilibrio inestable sin que en ninguna sección del soporte se alcance el agotamiento;

$k=3$ si la armadura es aquélla para la cual el soporte, bajo la solicitación de cálculo, alcanza un estado límite último de agotamiento, es decir, un estado de equilibrio estable pero en el que la sección crítica, bajo los efectos calculados en teoría de segundo orden, alcanza su agotamiento resistente.

El valor de $U$ es la capacidad mecánica teórica estricta de cada barra de la armadura, en toneladas. El valor de $\varnothing$ es el diámetro teórico correspondiente, en milímetros.

Una vez facilitados estos resultados, el programa vuelve atrás automáticamente, pidiendo un nuevo código de tarea $\mathrm{T}$.

En caso de existir varias hipótesis de cálculo o, más precisamente, varias solicitaciones actuantes sobre la misma sección, bastará con dar $T=5$ e introducir la siguiente solicitación $M_{d}, N_{d}$, obteniéndose la capacidad mecánica y el diámetro teórico necesarios para cada barra, y así sucesivamente. La sección deberá armarse para la mayor de las capacidades y diámetros obtenidos, que corresponderán a la hipótesis más desfavorable.

Si la capacidad o el diámetro resultantes aconsejan cambiar la disposición de armado elegida, ello podrá hacerse sin más que introducir $\mathrm{T}=4$, tecleando a continuación el código de armado CA de la nueva disposición y la solicitación o solicitaciones actuantes $M_{d}, N_{d}$.
Si se desea modificar el recubrimiento $r$ a la vista del diámetro normalizado resultante, bastará para ello con dar $\mathrm{T}=3$, introduciendo a continuación el nuevo recubrimiento $r$, y luego el código de armado CA y la solicitación o solicitaciones actuantes $M_{d}, N_{d}$.

Si se desea pasar a otro elemento con distinta sección o con distinta longitud de pandeo, bastará con $\operatorname{dar} \mathrm{T}=2$, introduciendo sucesivamente las dimensiones $b, h, l_{0}$, el recubrimiento $r$, el código de armado CA, y la solicitación o solicitaciones actuantes $M_{d}, N_{d}$.

Por último, si se desea cambiar la resistencia de cálculo del hormigón o del acero se dará $T=1$, introduciendo luego las nuevas resistencias $f_{c d}, f_{y d}$; a continuación las dimensiones del soporte $b, h$, $I_{0}$; el recubrimiento $r$; el código de armado CA, y la solicitación o solicitaciones actuantes $M_{d}, N_{d}$. El programa mantendrá las nuevas resistencias para el futuro - incluso aunque se le apaguehasta que sea arrancado de nuevo con RUN, en cuyo momento volverá a las habituales (si se le da $T=2$ ) o a otras nuevas (si se le da $T=1$ ).

El programa prosigue estos ciclos de forma indefinida. Si se desea interrumpirlo basta con pulsar la tecla ATTN cuando el programa está en espera de datos (por ejemplo, cuando pide un nuevo código de tarea T).

Si se ha cometido un error, o bien para hacer continuar el programa interrumpido mediante la tecla ATTN o al volver a encender el ordenador,

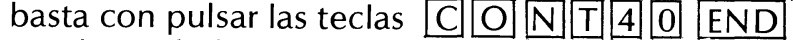
con lo cual el programa vuelve a pedir un nuevo código de tarea T, y prosigue su ejecución.

Como es obvio, al arrancar el programa con RUN hay que dar un código de tarea $T=1$ ó $\mathrm{T}=2$, pues si no el ordenador no dispondrá de todos los datos necesarios para el cálculo.

\section{Método de resolución}

Entre los métodos aproximados existentes para el cálculo de soportes esbeltos se ha elegido el Método de las Curvaturas de Referencia, MCR debido a Hugo Corres (2). Se trata de un método originalmente ideado para su aplicación gráfica (para lo que es preciso disponer previamente de colecciones de nomogramas) o mediante fórmulas aproximadas (válidas para casos particulares de distribución de armaduras, recubrimiento, límite elástico, etc.). Su aplicación numérica, desarrollada en este programa, tiene las siguientes ventajas:

- Tiempos de cálculo, para el dimensionamiento, sustancialmente más breves (un orden de magnitud) que los de otros métodos 
aproximados comparables, como el Método de la Columna Modelo, MCM, o el Método de la Deformada Senoidal, MDS.

- Errores muy pequeños y que tienden a quedar del lado de la seguridad. Referidos a axiles resistidos con una excentricidad constante, los errores medios son menores del $1 \%$, con desviaciones típicas del orden del 1,5\% (2). Los mayores errores se producen para pequeñas excentricidades y esbelteces elevadas.

- Facilidad de considerar el caso general en cuanto a distribución de armaduras, recubrimiento, límite elástico, etcétera.

- Posibilidad de distinguir claramente entre los dos estados límites últimos posibles en un soporte esbelto calculado en teoría de segundo orden: el de inestabilidad y el de agotamiento (ver apartado anterior).

Como es sabido, en el MCM, adoptado por el CEB (3), el comportamiento no lineal de la sección (no linealidad mecánica o del material) queda caracterizado por las llamadas directrices mecánicas (fig. 4), que son, en unos ejes excentricidades-curvaturas, los diagramas momento-curvatura, mientras que el comportamiento no lineal del soporte (no linealidad geométrica o estructural) queda caracterizado por la llamada directriz geométrica, que es una recta cuya ordenada en el origen es la excentricidad de primer orden $\eta_{\mathrm{o}}$ y cuya

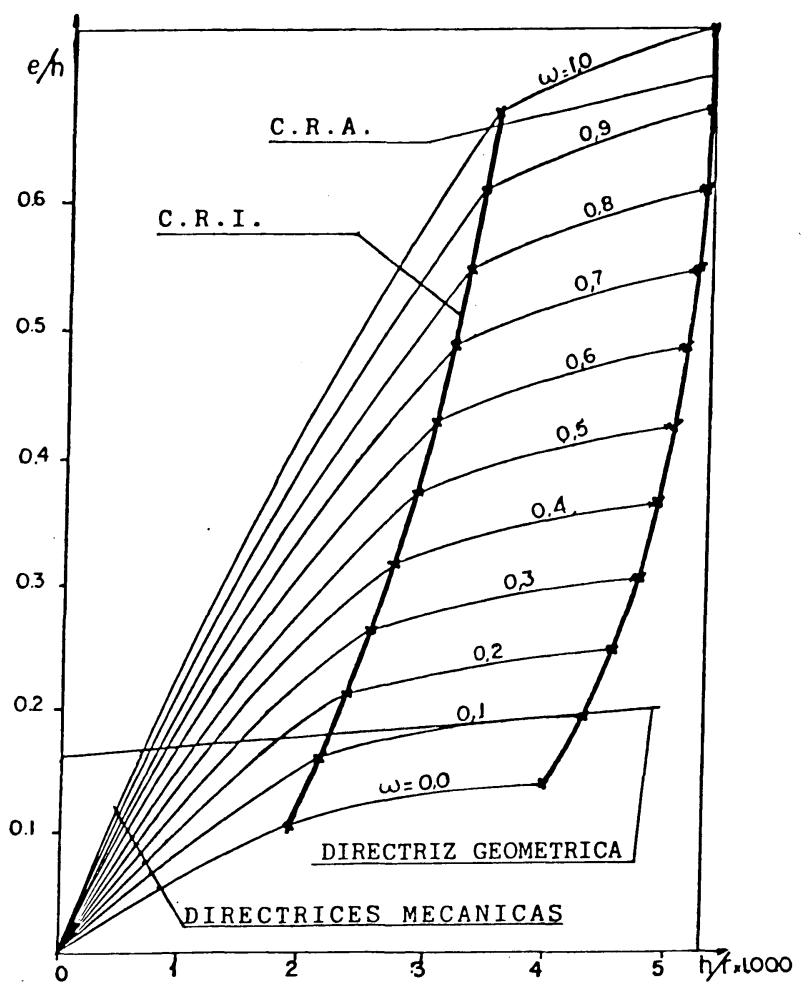

Fig. 4.-Método Columna Modelo y Método Curvaturas de Referencia pendiente es proporcional al cuadrado de la esbeltez, $\lambda^{2}$. Dado el axil reducido $v$ es posible construir una familia de directrices mecánicas para distintos valores de la cuantía mecánica de la armadura $\omega$, y conociendo $\eta_{\text {o }}$ y $\lambda$ se construye la directriz geométrica. El dimensionamiento estricto del soporte se consigue determinando la cuantía $\omega$ de la directriz mecánica tangente a la directriz geométrica (caso de inestabilidad), o bien, para excentricidades altas y esbelteces bajas, la de la directriz mecánica que es cortada por la directriz geométrica en su punto final (caso de agotamiento).

Pues bien, el MCR es una simplificación del MCM consistente en suponer que el punto de tangencia de las directrices mecánicas es precisamente el punto anguloso de las mismas (ver fig. 4), lo que es cierto en muchas ocasiones, y cuando no lo es conduce a dimensionamientos del lado de la seguridad. En vez de ser necesaria la obtención de toda la familia de directrices mecánicas, basta con obtener dos curvas graduadas en $\omega$ : la curva de curvaturas de referencia de inestabilidad (CRI), que contiene todos los puntos angulosos, y la curva de curvaturas de referencia de agotamiento (CRA), que contiene todos los puntos finales. El dimensionamiento del soporte se consigue encontrando la intersección de dichas curvas con la directriz geométrica, y leyendo los valores de $\omega$ correspondientes. El menor de estos dos valores de $\omega$ es la cuantía estricta necesaria para el soporte.

\section{Formulación}

a) El programa calcula, ante todo, la cuantía mecánica mínima de acuerdo con el apartado 38.3 de la Instrucción EH-82 (1):

$$
\omega_{m}=\rho_{m} \frac{f_{y d}}{f_{c d}}=0,004 \frac{f_{y d}}{f_{c d}}
$$

b) A continuación el programa calcula la cuantía mecánica de inestabilidad, $\omega_{i}$, que es la cuantía del punto de intersección de la curva CRI y de la directriz geométrica, según se ha visto en el apartado 3 (ver fig. 5).

Para ello sigue el método iterativo de la cuerda o de la regula falsi. Parte de los siguientes valores iniciales:

$$
\begin{aligned}
& \omega_{1}=1 ; \\
& \omega_{2}=v-0,8 \nless 0,2 .
\end{aligned}
$$

Entrando en la subrutina CRI (ver apartado siguiente) obtiene las coordenadas curvaturaexcentricidad de los puntos 1 y 2 (fig. 5). 


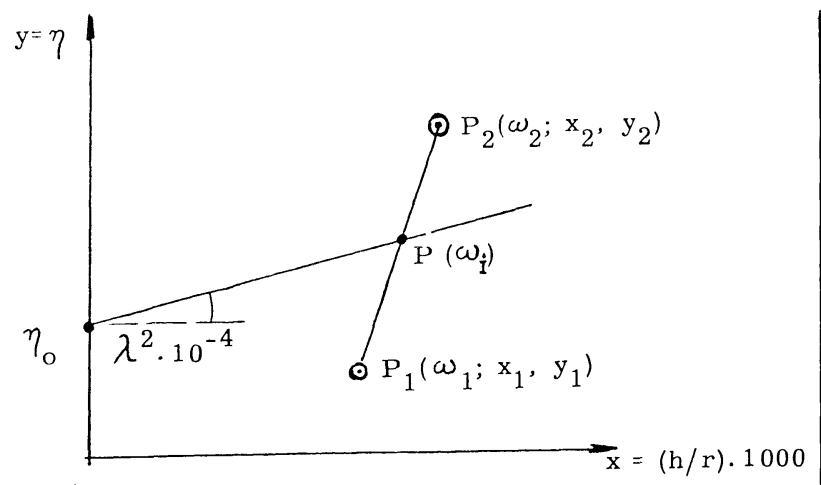

Fig. 5.-Subrutina CRI.

La cuantía del punto de intersección P buscado puede aproximarse mediante una interpolación lineal:

$$
\omega=\omega_{1}+\Delta \omega,
$$

siendo: $\triangle \omega=\left(\eta_{\mathrm{o}}+\mathrm{cx}_{1}-\mathrm{y}_{1}\right) /(\mathrm{b}-\mathrm{ac})$;

$$
\begin{aligned}
& \mathrm{a}=\left(\mathrm{x}_{2}-\mathrm{x}_{1}\right) /\left(\omega_{2}-\omega_{1}\right) ; \\
& \mathrm{b}=\left(\mathrm{y}_{2}-\mathrm{y}_{1}\right) /\left(\omega_{2}-\omega_{1}^{\prime}\right) ; \\
& \mathrm{c}=\lambda^{2} \cdot 10^{-4} .
\end{aligned}
$$

Se calcula la función error $\triangle^{\prime} \omega=\omega_{2}-\omega_{1}-\Delta \omega$. Si resultara $\left|\Delta^{\prime} \omega\right| \leq 0,001$ se da el proceso por terminado, pasando al punto siguiente c. En caso contrario, se hace:

$$
\begin{aligned}
& \omega_{1} \leftarrow \omega_{2} ; \\
& \left(x_{1}, y_{1}\right) \leftarrow\left(x_{2}, y_{2}\right) ; \\
& \omega_{2} \leftarrow \omega_{2}-\Delta^{\prime} \omega
\end{aligned}
$$

y se prosigue el proceso.

c) De igual forma se opera para el cálculo de la cuantía mecánica de agotamiento, $\omega_{\mathrm{a}}$, que es la cuantía del punto de intersección de la directriz geométrica con la curva CRA, entrando para ello en la subrutina CRA que calcula las coordenadas curvatura-excentricidad de un punto de dicha curva dado por su cuantía $\omega$ y por su axil reducido $v$.

d) La cuantía final de dimensionamiento es:

$$
\omega=\max \left[\omega_{\mathrm{m}}, \min \left(\omega_{\mathrm{i}}, \omega_{\mathrm{a}}\right)\right]
$$

\section{Subrutinas auxiliares}

A continuación se describen sucintamente las subrutinas del programa.

\subsection{Subrutina CRI}

Esta subrutina proporciona los valores de la curvatura $h / r$ y de la excentricidad relativa $\eta=\mathrm{e} / \mathrm{h}$ de un punto de la curva CRI, a partir de los valores del axil reducido $v=N_{d} /\left(f_{c d} \cdot b \cdot h\right)$ y de la cuantía mecánica total $\omega=A_{\text {tot }} \cdot f_{y d} /\left(f_{c d} \cdot b \cdot h\right)$.

Los puntos de CRI son aquellos en que la pendiente del diagrama momento-curvatura (directrices mecánicas) tiene un cambio brusco (puntos angulosos). Una observación de las directrices mecánicas (2) muestra que esos puntos se producen para posiciones del plano de deformaciones en las que una de las armaduras principales alcanza la deformación del límite elástico, $\varepsilon_{y}=f_{y d} / E_{s}$. Para axiles bajos $(v \leq 0,3)$, cuando la armadura inferior alcanza el límite elástico en tracción, $\varepsilon_{\mathrm{s}}=-\varepsilon_{\mathrm{y}}$. Para axiles medios y altos $(v>0,3)$, cuando la armadura superior alcanza el límite elástico en compresión, $\varepsilon_{\mathrm{s}}=\varepsilon_{\mathrm{y}}$.

Consecuentemente con lo anterior, la subrutina hace lo siguiente:

a) Si $v \leq 0,3$, explora las posiciones del plano de deformaciones con el pivote 2 indicado en la figura 6, hasta encontrar una para la cual el axil interno sea igual al axil externo dato $v$. Esto se hace por el método de la cuerda o de la regula falsi, partiendo de los valores iniciales:

$$
\begin{aligned}
& \mathrm{x}_{1}=\varepsilon_{\mathrm{c} 1}=0,001 \\
& \mathrm{x}_{2}=\varepsilon_{\mathrm{c} 2}=0,002
\end{aligned}
$$

Para cada valor de $\varepsilon_{c}$, conocidas las deformaciones en la fibra superior del hormigón y en la armadura inferior, $\varepsilon_{\mathrm{C}}$ y $\varepsilon_{5}$, se llama a la subrutina REVAC, que calcula el axil interno $v_{\mathrm{i}}$ y el momento interno $\mu_{\mathrm{i}}$, y se calcula el error, dado por la función error $\mathrm{y}=v_{\mathrm{i}}-v$.

b) Si se tiene $\left|y_{2}\right| \leq 0,0001$, se da el proceso por terminado. Los valores de la curvatura y de la excentricidad buscados son:

$$
\begin{aligned}
& \mathrm{h} / \mathrm{r}=\left(\varepsilon_{\mathrm{c}}-\varepsilon_{\mathrm{g}}\right) / \delta ; \\
& \eta=\mu_{\mathrm{i}} / \nu_{\mathrm{i}}
\end{aligned}
$$

siendo: $\delta=\mathrm{d} / \mathrm{h}$ el canto útil relativo;

$$
\begin{gathered}
\mu_{i}, \nu_{i} \text { el momento y axil reducidos } \\
\text { internos, resistidos por la sección. }
\end{gathered}
$$

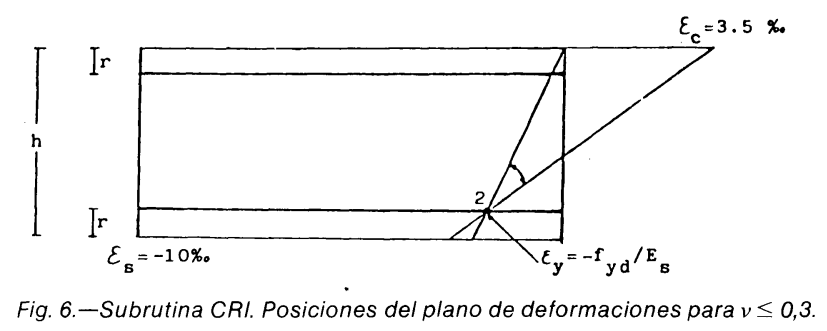


c) En caso contrario, a partir de los errores $y_{1}, y_{2}$ correspondientes a $x_{1}, x_{2}$ se calcula un nuevo valor de $\mathrm{x}=\varepsilon_{\mathrm{C}}$ :

$$
x=x_{2}-\Delta x \quad \text { con } \quad \Delta x=\frac{x_{2}-x_{1}}{y_{2}-y_{1}} y_{2}
$$

d) Se hace:

$$
\begin{aligned}
& x_{1} \leftarrow x_{2} ; \\
& y_{1} \leftarrow y_{2} ; \\
& x_{2} \leftarrow x_{2}-\Delta x
\end{aligned}
$$

y se prosigue el proceso.

e) En caso de que sea $v>0,3$, se comprueba que el axil $v$ es menor del que podría resistirse en compresión simple con $\varepsilon_{\mathrm{C}}=\varepsilon_{\mathrm{S}}=\varepsilon_{\mathrm{y}}$. Si no es así, se imprime el mensaje "AXIL EXCESIVO», y se detiene el proceso.

f) Si el axil no es excesivo, se exploran las posiciones del plano de deformaciones con el pivote 1 indicado en la figura 7 , hasta encontrar una para la que el axil interno sea igual al axil externo dato $v$.

Esto se hace como en los apartados a) a d) anteriores, pero variando $\varepsilon_{\mathrm{s}}$ (deformación en la armadura inferior). Se toman como valores iniciales:

$$
\begin{aligned}
& \mathrm{x}_{1}=\varepsilon_{\mathrm{s} 1}=-\varepsilon_{\mathrm{y}} \\
& \mathrm{x}_{2}=\varepsilon_{\mathrm{s} 2}=\varepsilon_{\mathrm{y}} .
\end{aligned}
$$

Para cada valor de $\varepsilon_{\mathrm{s}}$ se calcula la deformación en la fibra superior del hormigón $\varepsilon_{\mathrm{c}}$ mediante la condición del pivote 1 :

$$
\varepsilon_{\mathrm{C}}=\left(\delta \cdot \varepsilon_{\mathrm{y}}-\delta^{\prime} \cdot \varepsilon_{\mathrm{s}}\right) /\left(\delta-\delta^{\prime}\right),
$$

siendo:

$$
\begin{aligned}
& \delta=\mathrm{d} / \mathrm{h} \text { el canto útil relativo; } \\
& \delta^{\prime}=1-\delta \text { el recubrimiento relativo. }
\end{aligned}
$$

Conocidos $\varepsilon_{\mathrm{C}}$ y $\varepsilon_{\mathrm{s}}$ se entra en la subrutina REVAC y se prosigue el proceso hasta ajustar el axil.

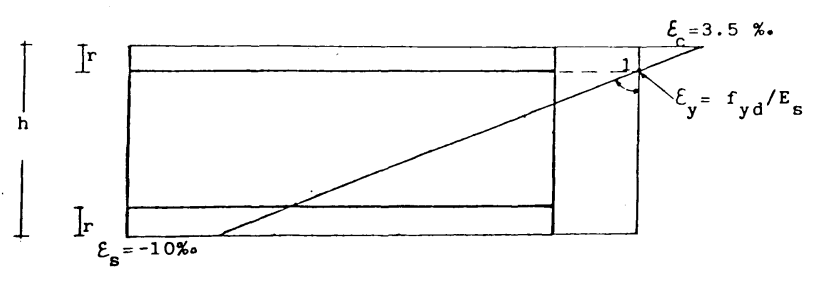

Fig. 7.-Subrutina CRI. Posiciones del plano de deformaciones para $v>0,3$.

\subsection{Subrutina $C R A$}

Esta subrutina, análoga a la CRI, proporciona los valores de la curvatura $h / r$ y de la excentricidad relativa $\eta=\mathrm{e} / \mathrm{h}$ de un punto de la curva CRA, a partir de los valores del axil reducido $v$ y de la cuantía mecánica $\omega$.

Los puntos de CRA son los puntos finales del diagrama momento-curvatura (directrices mecánicas), para los que la sección del soporte alcanza su agotamiento. Corresponden, por consiguiente, a posiciones del plano de deformaciones pertenecientes al diagrama de los pivotes de la figura 8 (Instrucción EH-82, apartado 36.2).

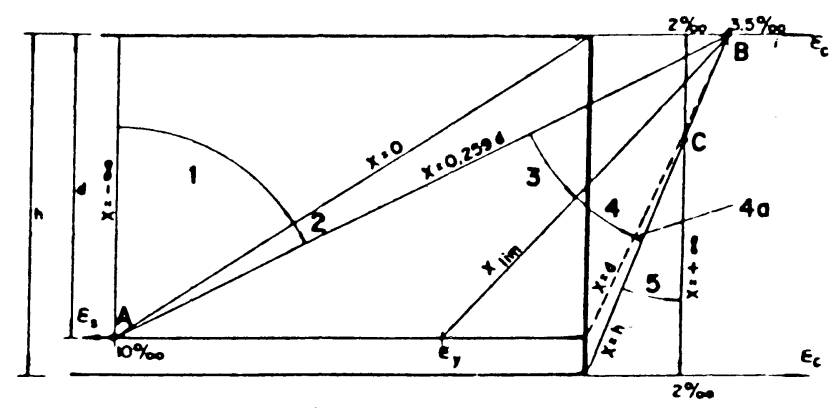

Fig. 8.-Subrutina CRA. Posiciones del plano de deformaciones (diagrama de pivotes).

La subrutina toma como parámetro la profundidad del eje neutro de deformaciones $\xi=x / h$. Dado un valor de $\xi$, la subrutina auxiliar DEFOR le proporciona los valores de la deformación en la fibra superior del hormigón, $\varepsilon_{\mathrm{c}}$, y en la armadura inferior, $\varepsilon_{s}$, de acuerdo con el diagrama de los pivotes. Posteriormente, la subrutina REVAC calcula el axil y el momento internos resistidos, $v_{i}, \mu_{i}$, que corresponderán a una situación de agotamiento.

El proceso seguido es el siguiente:

a) Se toman como valores iniciales para los tanteos los siguientes:

$$
\xi_{1}=0,5 ; \quad \xi_{2}=0,8
$$

b) Para cada uno de estos valores se calculan las deformaciones $\varepsilon_{\mathrm{c}}, \varepsilon_{\mathrm{s}}$ (subrutina DEFOR) y los esfuerzos $\nu_{i}, \mu_{i}$ (subrutina REVAC), y se evalúa la función de error $\mathrm{y}=v_{\mathrm{i}}-v$.

c) Si fuera $\left|y_{2}\right| \leq 0,0001$ se daría el proceso por terminado. Los valores de la curvatura y de la excentricidad buscados serían:

$$
\begin{aligned}
& \mathrm{h} / \mathrm{r}=\left(\varepsilon_{\mathrm{c}}-\varepsilon_{\mathrm{s}}\right) / \delta \\
& \eta=\mu_{\mathrm{i}} / \nu_{\mathrm{i}}
\end{aligned}
$$

(ver notaciones en el apartado 5.1). 
d) En caso contrario, a partir de los errores $y_{1}, y_{2}$ correspondientes a $\xi_{1}, \xi_{2}$ se calcula por el método de la cuerda un nuevo valor de $\xi$ :

$\xi=\xi_{2}-\Delta \xi$ con $\Delta \xi=\left(\xi_{2}-\xi_{1}\right) \cdot \mathrm{y}_{2} /\left(\mathrm{y}_{2}-\mathrm{y}_{1}\right)$

e) Se hace:

$$
\begin{aligned}
& \xi_{1} \leftarrow \xi_{2} ; \\
& y_{1} \leftarrow y_{2} ; \\
& \xi_{2} \leftarrow \xi_{2}-\Delta \xi,
\end{aligned}
$$

y se prosigue el proceso hasta ajustar el axil.

\subsection{Subrutina DEFOR}

Esta subrutina, auxiliar de la CRA, calcula los valores de las deformaciones en la fibra superior del hormigón, $\boldsymbol{\varepsilon}_{\mathrm{c}}$, y en la armadura inferior, $\varepsilon_{\mathrm{s}}$, correspondientes a una situación de agotamiento (diagrama de los pivotes de la fig. 8), en función de la profundidad relativa del eje neutro $\xi=\mathrm{x} / \mathrm{h}$. Las expresiones empleadas son las siguientes:

Dominio 2 (flexión compuesta): $0 \leq \xi<0,259 \cdot \delta$ :

$$
\begin{aligned}
& \varepsilon_{\mathrm{c}}=0,01 \cdot \xi /(\delta-\xi) ; \\
& \varepsilon_{\mathrm{s}}=-0,01 .
\end{aligned}
$$

Dominios 3 y 4 (flexión compuesta): $0,259 \delta \leq \xi<1$ :

$$
\begin{aligned}
& \varepsilon_{\mathrm{c}}=0,0035 ; \\
& \varepsilon_{\mathrm{s}}=0,0035(\xi-\delta) / \xi .
\end{aligned}
$$

Dominio 5 (compresión compuesta): $1 \leq \xi<2$ :

(En el dominio 5 la definición del parámetro $\xi$ ya no es $\xi=x / h$, pues ello daría valores muy grandes de $\xi$ en las proximidades de la compresión simple. El parámetro $\xi$ se hace variar entre 1 y 2 linealmente $\operatorname{con} \varepsilon_{\mathrm{c}}$ ).

$$
\begin{gathered}
\varepsilon_{\mathrm{c}}=0,0050-0,0015 \xi ; \\
\varepsilon_{\mathrm{s}}=\varepsilon_{\mathrm{r}}+(\xi-1)\left(0,0020-\varepsilon_{\mathrm{\vartheta}}\right),
\end{gathered}
$$

siendo: $\varepsilon_{\mathrm{r}}=0,0035(1-\delta)$ la deformación en la armadura inferior para $\xi=1$.

\subsection{Subrutina REVAC}

Esta subrutina, que es Ilamada por la CRI y por la CRA, calcula los valores de los esfuerzos internos $\nu_{i}, \mu_{i}$ a partir de las deformaciones en la fibra superior del hormigón $\varepsilon_{\mathrm{c}}$ y en la armadura inferior $\varepsilon_{s}$. El proceso seguido es el que se indica a continuación: a) Primeramente la subrutina comprueba que las deformaciones $\varepsilon_{\mathrm{c}}$ y $\varepsilon_{\mathrm{s}}$ datos no constituyen una situación correspondiente a un estado posterior al agotamiento, o sea, que no se rebasa ninguno de los tres pivotes del diagrama de la figura 8:

Pivote A: $\varepsilon_{\mathrm{s}} \geq-0,01$;

Pivote B: $\varepsilon_{\mathrm{c}} \leq 0,0035$;

Pivote C: $\left[3 / 7 \cdot \varepsilon_{\mathrm{s}}+\left(4 / 7-\delta^{\prime}\right) \varepsilon_{\mathrm{c}}\right] / \delta \leq 0,002$.

También se comprueba que la curvatura es positiva:

$$
\varepsilon_{\mathrm{C}} \geq \varepsilon_{\mathrm{s}}
$$

En caso de que una cualquiera de estas cuatro condiciones no se cumpliera, el programa se detiene, apareciendo en pantalla el mensaje «ERROR EN SUBR. REVAC».

b) A continuación se obtiene la profundidad del eje neutro $\xi$ :

$$
\xi=\frac{\varepsilon_{\mathrm{C}}}{\varepsilon_{\mathrm{c}}-\varepsilon_{\mathrm{s}}} \cdot \delta
$$

c) Luego se calculan la resultante de las tensiones del hormigón $v_{c}$ y el momento resultante $\mu_{\text {co }}$ con respecto al eje neutro, para lo cual se distinguen dos casos:

c1) Caso $\xi \leq 1$ (flexión compuesta):

$$
\begin{gathered}
v_{\mathrm{c}}=\int_{\mathrm{o}}^{\xi} \sigma_{\mathrm{c}}\left(\varepsilon_{\mathrm{y}}\right) \mathrm{dy} ; \\
\mu_{\mathrm{co} .}=\int_{\mathrm{o}}^{\xi} \sigma_{\mathrm{c}}\left(\varepsilon_{\mathrm{y}}\right) \mathrm{y} \mathrm{dy},
\end{gathered}
$$

siendo $\varepsilon_{\mathrm{y}}=\varepsilon_{\mathrm{c}} \cdot \mathrm{y} / \xi$ la deformación a la distancia y del eje neutro.

c2) Caso $\xi>1$ (Compresión compuesta):

$$
\begin{aligned}
& \nu_{\mathrm{c}}=\int_{\mathrm{o}}^{\xi} \sigma_{\mathrm{c}}\left(\varepsilon_{\mathrm{y}}\right) \mathrm{dy}-\int_{\mathrm{o}}^{\xi-1} \sigma_{\mathrm{c}}\left(\varepsilon_{\mathrm{y}}\right) \mathrm{dy} ; \\
& \mu_{\mathrm{co}}=\int_{\mathrm{o}}^{\xi} \sigma_{\mathrm{c}}\left(\varepsilon_{\mathrm{y}}\right) \mathrm{ydy}-\int_{\mathrm{o}}^{\xi-1} \sigma_{\mathrm{c}}\left(\varepsilon_{\mathrm{y}}\right) \mathrm{ydy} .
\end{aligned}
$$

Estas integrales se calculan mediante Ilamadas a la subrutina auxiliar ESREV (apartado 5.5). En el caso c1 se efectúa una única Ilamada. En el caso c2 se efectúan dos Ilamadas, la primera con el argumento $\xi$ y la segunda con el argumento $\xi-1$. 
d) Se calcula el momento resultante del hormigón respecto al centro de la sección:

$$
\begin{aligned}
& y_{\mathrm{g}}=\mu_{\mathrm{co}} / \nu_{\mathrm{c}} ; \\
& \mu_{\mathrm{c}}=\nu_{\mathrm{c}}\left(\mathrm{y}_{\mathrm{g}}-\xi+0,5\right),
\end{aligned}
$$

siendo $y_{g}$ la distancia de la resultante del bloque de compresiones del hormigón al eje neutro.

f) A continuación se calculan los esfuerzos resistidos por las armaduras:

$$
\begin{aligned}
& v_{s}=\sum_{i=1}^{n_{2}} A_{i} \cdot \sigma_{s i}\left(\varepsilon_{u}\right) ; \\
& \mu_{s}=\sum_{i=1}^{n_{2}} A_{i} \cdot \sigma_{s i}\left(\varepsilon_{u}\right) \cdot u,
\end{aligned}
$$

siendo:

$$
\begin{aligned}
\mathrm{n}_{2}= & \text { número de capas de } \\
& \text { armadura = segunda cifra del } \\
& \text { código de armado CA; }
\end{aligned}
$$

$\mathrm{A}_{\mathrm{i}}=$ el área relativa de la capa de armadura i:

$A_{i}=n_{1} \cdot \omega / n_{\text {tot }}$ para las capas extremas $\left(i=1\right.$ e $\left.i=n_{2}\right)$;

$\mathrm{A}_{\mathrm{i}}=2 \cdot \omega / \mathrm{n}_{\text {tot }}$ para las restantes capas;

$\mathrm{n}_{1}=$ número de barras de las capas extremas $=$ primera cifra del código de armado CA;

$\omega=$ la cuantía mecánica total de la armadura;

$\sigma_{\mathrm{si}}=$ la tensión relativa de la armadura de la capa i:

$\sigma_{\mathrm{si}}=\varepsilon_{\mathrm{u}} / \varepsilon_{\mathrm{y}}$ con las limitaciones $\sigma_{\mathrm{si}} \ngtr 1$ y $\sigma_{\mathrm{si}} \nless-1$;

$\varepsilon_{\mathrm{u}}=$ la deformación de la armadura i;

$\varepsilon_{y}=\mathrm{f}_{\mathrm{yd}} / \mathrm{E}_{\mathrm{s}}$ la deformación de límite elástico del acero;

$\mathrm{u}=$ la ordenada de la capa i de armadura, respecto al centro de la sección;

$\mathrm{n}_{\text {tot }}=2\left(\mathrm{n}_{1}+\mathrm{n}_{2}-2\right)=$ número total de barras. g) Los esfuerzos totales se obtienen sumando las contribuciones del hormigón y de las armaduras:

$$
\begin{aligned}
& \nu_{\mathrm{i}}=\nu_{\mathrm{c}}+\nu_{\mathrm{s}} ; \\
& \mu_{\mathrm{i}}=\mu_{\mathrm{c}}+\mu_{\mathrm{s}} .
\end{aligned}
$$

\subsection{Subrutina ESREV}

Esta subrutina, esclava de la REVAC, calcula las integrales que dan la resultante y el momento del bloque de tensiones del hormigón. Para ello distingue dos casos:

a) Si se verifica $\varepsilon_{c} \leq 0,002$, el bloque de compresiones está todo él en la zona parabólica del diagrama parábola-rectángulo (no se llega a la zona rectangular).

Entonces:

$$
\sigma_{\mathrm{c}}\left(\varepsilon_{\mathrm{y}}\right)=850 \varepsilon_{\mathrm{y}}-212500 \varepsilon_{\mathrm{y}}^{2} ;
$$

pero: $\quad \varepsilon_{\mathrm{y}}=\varepsilon_{\mathrm{c}} \cdot \mathrm{y} / \xi$,

de donde: $\sigma_{c}\left(\varepsilon_{y}\right)=A \cdot y+B \cdot y^{2}$,

siendo: $A=850 \varepsilon_{\mathrm{c}} / \xi$;

$$
B=-212500\left(\varepsilon_{c} / \xi\right)^{2} \text {. }
$$

De aquí resulta:

$$
\begin{aligned}
& \mathrm{I}_{1}(\xi)=\int_{0}^{\xi} \sigma_{\mathrm{c}} \mathrm{dy}=\mathrm{A} \cdot \xi^{2} / 2+\mathrm{B} \cdot \xi^{3} / 3 ; \\
& \mathrm{I}_{2}(\xi)=\int_{0}^{\xi} \sigma_{\mathrm{c}} \mathrm{y} \mathrm{dy}=\mathrm{A} \cdot \xi^{3} / 3+\mathrm{B} \cdot \xi^{4} / 4 .
\end{aligned}
$$

b) Si se verifica $\varepsilon_{c}>0,002$, entonces hay parte del bloque en zona rectangular. En ese caso se calcula:

$$
\begin{aligned}
& \xi_{\mathrm{R}}=\left(0,002 / \varepsilon_{\mathrm{C}}\right) \cdot \xi ; \\
& \mathrm{I}_{1}(\xi)=\mathrm{I}_{1}\left(\xi_{\mathrm{R}}\right)+0,85\left(\xi-\xi_{\mathrm{R}}\right) ; \\
& \mathrm{I}_{2}(\xi)=\mathrm{I}_{2}\left(\xi_{\mathrm{R}}\right)+0,85\left(\xi-\xi_{\mathrm{R}}\right)\left(\xi+\xi_{\mathrm{R}}\right) / 2 .
\end{aligned}
$$

\section{Listado en BASIC}

Al final del artículo se incluye un listado del programa y de las subrutinas en el lenguaje BASIC del ordenador personal portátil HP-75. Este listado ha sido producido por la impresora portátil HP-82162A, que imprime líneas con un máximo de 24 caracteres; cuando una instrucción no cabe en una línea, la corta en los caracteres núm. 24, 48, etc., y la imprime en varias líneas sucesivas. 
El ordenador HP-75 es un modelo más antiguo de la serie del HP-71. El lenguaje BASIC de ambos es perfectamente compatible.

\section{Ejemplo}

Se trata de dimensionar un soporte esbelto de $\mathrm{b} \cdot \mathrm{h}=30 \cdot 40 \mathrm{~cm}^{2}$ de sección y una longitud de pandeo $\mathrm{I}_{\mathrm{o}}=6,00 \mathrm{~m}$ (véase fig. 2), sometido a las siguientes solicitaciones actuantes:

a) $M_{d}=14,9 \mathrm{mt} ; \mathrm{N}_{\mathrm{d}}=81,9 \mathrm{t}$.

b) $M_{d}=8,5 \mathrm{mt} ; N_{d}=105 \mathrm{t}$.

Se adoptan los siguientes valores de resistencias y coeficientes de seguridad:

$$
\begin{aligned}
\mathrm{f}_{\mathrm{ck}} & =200 \mathrm{kp} / \mathrm{cm}^{2} ; \\
\gamma_{\mathrm{c}} & =1,5 ; \\
\mathrm{f}_{\mathrm{cd}} & =200 \cdot 0,9 / 1,5=120 \mathrm{kp} / \mathrm{cm}^{2} ; \\
\mathrm{f}_{\mathrm{yk}} & =4.100 \mathrm{kp} / \mathrm{cm}^{2} ; \gamma_{\mathrm{s}}=1,1 ; \\
\mathrm{f}_{\mathrm{yd}} & =4.100 / 1,1=3.727 \mathrm{kp} / \mathrm{cm}^{2} .
\end{aligned}
$$

Suponiendo un recubrimiento $r=4 \mathrm{~cm}$ y la disposición de armado de la figura 2, los datos y resultados serán los que se pueden apreciar en el cuadro de pie de página, incluyendo el tiempo de cálculo en el ordenador HP-75 (en el HP-71 los tiempos son aproximadamente dobles*).

(*) El programa, con adaptaciones mínimas, ha corrido también en los ordenadores domésticos ZX SPECTRUM (precio actual: unas 20.000 ptas.) y AMSTRAD 464, con indénticos resultados y tiempos de cálculo iguales a los dados multiplicados por los factores 2,0 y 0,4 , respectivamente.
Como puede verse, se calcula a pandeo en las dos direcciones por separado. Para calcular en la dirección del ancho $b$, se intercambian entre sí las dimensiones de la sección $b$ y $h$ y se introduce un momento nulo. El programa toma como momento el correspondiente a la excentricidad mínima $\mathrm{e}_{\mathrm{o}}=2 \mathrm{~cm}$, de acuerdo con los apartados 36.3 y 43.2 .3 de la Instrucción EH-82 (1). La hipótesis en la que el esfuerzo axil es más pequeño $\left(\mathrm{N}_{\mathrm{d}}=81,9 \mathrm{t}\right)$ no es necesario calcularla en la dirección $b$, ya que conducirá necesariamente a una menor armadura.

El armado correcto de la sección resulta ser de $8 \varnothing 25$ (fig. 2 ).

\section{Referencias}

1. Instrucción EH-82 para el proyecto y la ejecución de obras de hormigón en masa o armado. Comisión Permanente del Hormigón. MOPU, 1982.

2. HUGO CORRES y FRANCISCO MORAN: «El método de las curvaturas de referencia». Monografía n. ${ }^{\circ} 370$ del Instituto Eduardo Torroja de la Construcción y del Cemento. Madrid, Junio 1982

3. CEB Design Manual on Buckling. The Construction Press. London, 1978.

4. FRANCISCO MORAN: "Programa para el dimensionamiento de secciones rectangulares de hormigón armado en flexocompresión recta con un ordenador portátil». Hormigón y Acero n. ${ }^{\circ} 153$, cuarto trimestre de 1984.

5. FRANCISCO MORAN: «Programa para la comprobación de secciones rectangulares de hormigón armado en flexocompresión esviada con un ordenador portátil». Informes de la Construcción 359-360, abril-mayo, 1984

6. FRANCISCO MORAN: «Programa para el dimensionamiento de secciones poligonales de hormigón armado en flexocompresión esviada con un ordenador portátil». Informes de la Construcción 363, agosto-septiembre, 1984 .

\begin{tabular}{|c|cc|ccc|c|c|cc|ccc|c|}
\hline Tarea & $\mathrm{f}_{\mathrm{cd}}$ & $\mathrm{f}_{\mathrm{yd}}$ & $\mathrm{b}$ & $\mathrm{h}$ & $\mathrm{l}_{\mathrm{o}}$ & $\mathrm{r}$ & $\mathrm{CA}$ & $\mathrm{M}_{\mathrm{d}}$ & $\mathrm{N}_{\mathrm{d}}$ & $\mathrm{k}$ & $\mathrm{U}$ & $\phi$ & $\mathrm{t}(\mathrm{seg})$ \\
\hline 1 & 120 & 3727 & 30 & 40 & 600 & 4 & 33 & 14.9 & 81.9 & 3 & 14.78 & 22.47 & 28 \\
5 & & & & & & & & 8.5 & 105 & 2 & 10.62 & 19.05 & 22 \\
2 & & & 40 & 30 & 600 & 4 & 33 & 0 & 105 & 2 & 5.39 & 13.57 & 26 \\
\hline
\end{tabular}




\section{LISTADO DEL PROGRAMA Y DE SUS SUBRUTINAS}

PROGRAMA DMNP

10 REK DHNP: DIMENS. SOP - PANDEO

28 DIH H(3)

$38 \mathrm{Fl}=1758 * .9 / 1.5$ \& $\mathrm{F}=4$ $.2 / 1.1$ e $\mathrm{E}=\mathrm{F} 2 / 2100$

40 INPUT 'Tare $a={ }^{\prime} ; K$

50 ON K GOTO $68,80,180,1$

18,178

60 INPUT ' $f c d, f y d(k p / c A 2$

)$=\cdot ; F 1, F 2$

$78 \mathrm{Fl}=\mathrm{F} 1 * 18$ \& $\mathrm{F} 2=\mathrm{F} 2 / 1808$

e $\mathrm{ES}=\mathrm{F} 2 / 2180$

80 INPUT ' $b, h, l o(C A)={ }^{\prime} ; B$

$, H, L$

$96 \mathrm{~B}=\mathrm{B} / 108 \mathrm{H}=\mathrm{H} / 100 \mathrm{~L}$ $=L / 100$

180 INPUT Recubr. $r\left(C_{n}\right)$

$=$; $C \in C=C / 100$

110 INPUT 'Cod. Arnado CA $=\cdot ;$ N2

$128 \mathrm{HL}=\mathrm{INT}(\mathrm{N} 2 / 10)$

$130 \mathrm{~N} 2=\mathrm{N} 2-10+\mathrm{N} 1$

$14 \theta \mathrm{R}=\mathrm{C} / \mathrm{H} \& \mathrm{D}=1-\mathrm{R}$

$15 \theta W(1)=4 \theta * F 2 / F 1$

$160 \mathrm{ET}=\mathrm{H} / 2 \mathrm{Q} \theta$ IF $\mathrm{E}<\mathrm{.02}$

480 REH SUBR. ESREY

496 IF $X=\theta$ THEN RETURH

$598 \mathrm{Al}=\mathrm{E} 1 / \mathrm{X}$

$516 \mathrm{~A} 2=\mathrm{A} 1 * \mathrm{~A} 1$

$520 \mathrm{Al}=859 * \mathrm{A1}$

$539 A z=-212599 * A 2$

638 REY SUBR. REYAC

648 IF E1).8835 OR E2〈-.

01 OR E1<E2-.089801 THEN 980

650 IF $(.4285 * E 2+(.5714-$

R)*E1)/D>.082 THEN 980

660 IF $A B S(E 1-E 2)<.00800$

1 THEN 788

$678 E 3=(E 1-E 2) / D$

$680 \mathrm{X}=\mathrm{E} 1 / \mathrm{E} 3$

$690 \mathrm{RI}=0$ \& R2=0

$780 \mathrm{~S}=1$ \& GOSUB 480

710 IF $X<=1$ THEN 750

$720 \mathrm{EI}=\mathrm{E} \mathrm{E} *(\mathrm{X}-1) / \mathrm{X} X \mathrm{X}=\mathrm{X}-$

1

$730 \mathrm{~S}=-1$ GOSUB 480

$748 X=X+1 \quad E 1=E 1 * X /(X-1$
THEN E7 $=.02$

170 INPUT ' $\operatorname{ld}, \operatorname{Hd}(\mathrm{At}, \mathrm{t})=$ '

; G2, G1

180 IF G2 $\angle E 7 * G 1$ THEN G2=

$E 7 *[1$

$199 \mathrm{G} 3=\mathrm{G} 2 / \mathrm{G} 1 / \mathrm{H}$

$20064=.8901 * \mathrm{~L} * \mathrm{~L} / \mathrm{H} / \mathrm{H}$

$210 \mathrm{Z}=\mathrm{G} 1 / \mathrm{F} 1 / \mathrm{B} / \mathrm{H}$

$228 \mathrm{Kl}=1 \in \mathrm{K} 2=\mathrm{Z}-.8 \mathrm{IF}$

$\mathrm{K} 2<.2$ THEN K2 $=.2$

238 H=K1 GOSUE 996

$240 \mathrm{PI}=\mathrm{F} \& \mathrm{Q1}=\mathrm{G}$

$250 \mathrm{H}=\mathrm{K2}$ e COSUB 990

$260 \mathrm{P2}=\mathrm{F}$ \& $Q 2=\mathrm{C}$

$278 P=(P 2-P 1) /(K 2-K 1) Q$

$Q=(Q 2-Q 1) /(K 2-K 1)$

$280 \quad K 3=K 2-K 1-(G 3+G 4 * P 1-Q$

1) $/(Q-P * G 4)$

290 IF $A B S(K 3)<=.801$ THE N 320

$300 \quad K 1=K 2$ \& $P 1=P 2$ Q $01=0$

2

$318 \mathrm{~K} 2=\mathrm{K} 2-\mathrm{K} 3 \mathrm{Q}$ G0T0 250

$32 \theta H(2)=K 2-K 3$

\section{SUBRUTINA ESREV}

$540 Y=X$

550 IF E1 $>.802$ THEH $Y=.0$

B2*X/E1

$568 X 2=Y * Y$

$570 Y 3=X 2 * Y / 3$

$580 \mathrm{~T} 1=\mathrm{A} 1 * \mathrm{X} 2 / 2+\mathrm{A} 2 * \mathrm{X} 3+.85$

SUBRU TINA REVAC

)

750 IF $X=0$ THEN 820

$760 R 2=R 1 *(R 2 / R 1-X+.5)$

770 GOTO 820

$780 \mathrm{R} 2=0 \quad \mathrm{E} 3=0$

790 IF $\mathrm{E} 1<=0$ THEN $\mathrm{R} 1=0 \mathrm{Q}$ GOTO 820

898 IF $E 1>=.092$ THEK $R 1=$

.85 G0T0 820

$810 R 1=(859-212580 * E 1) * E$

1

820 E4=E2 $Y Y=R-.5$ \& Y1= $2 * Y /(1-H 2)$

$830 \mathrm{~S} 1=0$ S2=6 $\mathrm{EG}=\mathrm{E} 3$ *

Y1

840 FOR I $=1$ TO N2

859 IF $E 4\rangle=E 5$ THEN $S=1$
$339 K 1=K 2$ e $P 1=P 2$ Q $Q 1=0$

2 e $K 2=K 2+.1$

$340 \mathrm{H}=\mathrm{K} 2$ e GOSUB 1230

$350 \mathrm{P2}=\mathrm{F}$ Q $Q 2=\mathrm{G}$

$360 P=(P 2-P 1) /(K 2-K 1)$

$Q=(Q 2-Q 1) /(K 2-K 1)$

$378 K 3=K 2-K 1-(G 3+G 4 \pm P 1-\theta$

1) $/(Q-P * G 4)$

380 IF $\mathrm{ABS}(\mathrm{K} 3)<=.001$ THE N 410

$390 \mathrm{KI}=\mathrm{K} 2 \mathrm{P} 1=\mathrm{P} 2 \quad \mathrm{Q1}=0$ 2

$489 K 2=K 2-K 3$ G GOTO 340

$416 H(3)=k 2-k 3$

420 IF $H(1)>H(2)$ OR $H(1)$ ᄀH(3) THEH I=1 GOTO 44 $\theta$

430 IF $H(2)<H(3)$ THEN I= 2 ELSE $I=3$

$448 \mathrm{U}=\mathrm{H}(\mathrm{I}) * \mathrm{~F} 1 * \mathrm{~B} \neq \mathrm{H} / \mathrm{H} 1+\mathrm{H} 2$ $-2) / 2$

$458 \mathrm{~F} 4=\mathrm{SQR}(4 \mathrm{GG}+\mathrm{U} / \mathrm{F} 2 / \mathrm{PI})$

460 DISP USING $20,2(5 \mathrm{D}$.

2D) ; I,U,F4

470 HคIT 8 GOTO 40

$*(X-Y)$

$598 \quad \mathrm{~T} 2=\mathrm{A} 1 * X 3+\mathrm{A} 2 * X 3 * Y * .75$

$+.425 *(X-Y) *(X+Y)$

$600 \mathrm{R} 1=\mathrm{R} 1+5 * \mathrm{~T} 1$

$610 \mathrm{R} 2=\mathrm{R} 2 \mathrm{R}+\mathrm{S} * \mathrm{~T} 2$

620 RETURH

G0T0 880

868 IF $E 4<=-E 5$ THEN $S=-1$

e G0T0 880

$870 \mathrm{~S}=\mathrm{E} 4 / \mathrm{ES}$

880 IF I=1 OR I=N2 THEN

$S=N 1 \neq S$ ELSE $S=2 \neq S$

$890 \mathrm{S1}=\mathrm{S1}+\mathrm{S}$

$980 \mathrm{~S} 2=\mathrm{S} 2+\mathrm{S}+\mathrm{Y}$

$910 E 4=E 4+E 6$

$920 Y=Y+Y 1$

930 NEXT I

$946 \mathrm{~S}=\mathrm{H} / 2 /(\mathrm{H} 1+\mathrm{H} 2-2)$

$950 \mathrm{R} 1=\mathrm{R} 1+\mathrm{S} 1 * \mathrm{~S}$

$960 \mathrm{R} 2=\mathrm{R} 2+\mathrm{S} 2 * \mathrm{~S}$

970 RETURH

980 DISP -ERROR EN SUBR.

REYAC- $\mathrm{STOP}$ 
SUBRU TINA CRI

990 REM SUBR CRI

1809 IF $2>.3$ THEN 1898

$1810 \mathrm{E2}=-\mathrm{E5}$

$1828 \mathrm{Cl}=.081$ e $\mathrm{C} 2=.082$

$1830 \mathrm{EL}=\mathrm{C1}$ COSUB 630 ?

$D 1=R 1-Z$

$1048 \mathrm{E1}=\mathrm{C2}$ GOSUB $630 \mathrm{e}$

$\mathrm{D} 2=\mathrm{R} 1-\mathrm{Z}$

1850 IF ABS $(\mathrm{D} 2)<=.0801 \mathrm{~T}$

HEN 1208

$1068 \mathrm{~B} 3=(\mathrm{C} 1-\mathrm{C} 2) /(\mathrm{D} 1-\mathrm{D} 2) *$

D2

1230 REM SUBR CRA

$1248 \mathrm{Cl}=.5$ \& $\mathrm{C2}=.8$

$1250 x=c 1$ e GOSUB 1330 e

COSUB 638 \& $D 1=R 1-Z$

$1268 x=C 2$ e GOSUB 1330 e
$1870 \mathrm{Cl}=\mathrm{C} 2$ e $\mathrm{D1}=\mathrm{D} 2 \mathrm{C2}=$

$\mathrm{C} 2-\mathrm{D} 3$

1680 GOTO 1840

$1098 \mathrm{El}=\mathrm{ES}$ E2=E5 \& $\mathrm{COS}$ UB 638

1180 IF $2>R 1$ THEN DISP .

AXIL EXCESIYO STOP

$1110 \mathrm{Cl}=-\mathrm{E} 5$ \& $\mathrm{C} 2=\mathrm{E} 5$

$1120 \quad E 2=C 1$ E1 $=(D * E 5-R *$

$E 2) /(D-R)$

1130 GOSUB 630 \&I=R1-Z

$1140 \mathrm{E} 2=\mathrm{C} 2$ \& $\mathrm{E} 1=(\mathrm{II} * \mathrm{E} 5-\mathrm{R} *$

\section{SUBRUTINA CRA}

\section{GOSUB 638 - D2=R1-2}

1270 IF $\mathrm{ABS}(\mathrm{D} 2)<=.0001 \mathrm{~T}$

HEN 1380

$1288 \mathrm{D} 3=(\mathrm{C} 1-\mathrm{C} 2) /(\mathrm{D} 1-\mathrm{D} 2) *$

D2
$E 2) /(D-R)$

1150 GOSUB $630 \& \mathrm{D} 2=\mathrm{R} 1-\mathrm{Z}$

1160 IF ABS $(D 2)<=.8801 \mathrm{~T}$

HEN 1290

$1170 \mathrm{~B}=(\mathrm{C} 1-\mathrm{C} 2) /(\mathrm{D1} 1-\mathrm{D} 2) *$

D2

$1180 \mathrm{Cl}=\mathrm{C} 2 \mathrm{D1}=\mathrm{D} 2 \mathrm{C} \mathrm{C}=$ $\mathrm{C} 2-\mathrm{DB}$

1198 GOTO 1140

$1280 \mathrm{G}=\mathrm{R} 2 / \mathrm{R} 1$

$1210 \mathrm{~F}=(\mathrm{E} 1-\mathrm{E} 2) / \mathrm{D} * 1868$

1220 RETURH

\section{SUBRUTINA DEFOR}

1330 REM SUBR DEFOR

1348 IF $X>259 * D$ THEN 13

60

$1358 E 1=.01 * X /(D-X) \subseteq E 2$
$=-.01$ RETURN

1360 IF $X>1$ THEN 1380

$1370 \mathrm{E} 1=.0835$ e $E 2=.0935$

$\$(X-D) / X$ \& RETURH
$1290 \mathrm{Cl} 1=\mathrm{C} 2 \mathrm{DI}=\mathrm{D} 2 \mathrm{C2}=$ C2-D3 e GOTO 1260

$1390 \mathrm{G}=\mathrm{R} 2 / \mathrm{R} 1$

$1310 \mathrm{~F}=(\mathrm{E} 1-\mathrm{E} 2) / \mathrm{D}) 1000$

1320 RETURH

$$
\text { is is is }
$$

$1380 \mathrm{El}=.805-.0815 * \mathrm{X} \mathrm{E}$ $2=.6935 * \mathrm{R}$

$1390 \mathrm{E} 2=\mathrm{E} 2+(\mathrm{X}-1) *(.892-\mathrm{E} 2)$

1489 RETURH

\section{publicación del i.e.t.c.c.}

\begin{tabular}{|c|c|}
\hline $\begin{array}{l}\text { código-modelo ceb-fip } \\
\text { para las estructuras de hormigón }\end{array}$ & $\begin{array}{l}\text { El Instituto Eduardo Torroja, miembro activo tanto del Comité Eurointernacional del Hormigón (CEB), como de la } \\
\text { Federación Internacional del Pretensado (FIP), ha tomado a su cargo la traducción y edición de esta importante } \\
\text { normativa. } \\
\text { Aunque presentado con el título de "Código Modelo CEB/FIP 1978» este documento incorpora los dos primeros } \\
\text { volúmenes de este "Sistema Unificado Internacional de Reglamentación Técnica de Ingeniería Civil». El primer } \\
\text { volumen de este «Sistema Unificado" es el denominado "Reglas comunes Unificadas para los diferentes tipos de } \\
\text { obras y materiales», donde se exponen los criterios y formatos de seguridad a que han de ajustarse los diferentes } \\
\text { Códigos (estructuras de hormigón, estructuras metálicas, estructuras mixtas, estructuras de albañilería y estructuras } \\
\text { de madera), que han de configurar la totalidad del antedicho sistema. } \\
\text { El segundo volumen es propiamente el Código Modelo para las Estructuras de Hormigón. Fruto de la colaboración } \\
\text { de dos asociaciones del prestigio del CEB y la FIP, desde mediados de los } 60 \text {, incorpora los avances cientificos } \\
\text { y tecnológicos producidos en los últimos años sin detrimento alguno de la claridad y operatividad que deben } \\
\text { presidir un código que pretende ser, ante todo, un auxiliar práctico para los técnicos de la construcción. } \\
\text { El Código sigue en su estructura las reglas más o menos clásicas: una primera parte dedicada a los datos } \\
\text { generales para el cálculo (propiedades de los materiales, datos relativos al pretensado, tolerancias); en segundo } \\
\text { lugar se presentan las reglas de proyecto estructural lacciones, solicitaciones, estados limites últimos y de } \\
\text { utilización, reglas de detalle para el armado); y, por último, ejecución, mantenimiento y control de calidad. } \\
\text { También incluye reglas para estructuras con elementos prefabricados y estructuras de hormigón con áridos ligeros. } \\
\text { Los Anejos del Código se refieren a: terminología, proyecto mediante la experimentación, resistencia al fuego, } \\
\text { tecnología del hormigón, comportamiento en el tiempo del hormigón y fatiga. } \\
\text { Un volumen encuadernado en cartoné, de } 21 \times 30 \mathrm{~cm} \text {, compuesto de } 340 \text { páginas, Madrid, mayo } 1982 \text {. } \\
\text { Precios: España } 2.500 \text { ptas. Extranjero } 36 \$ \text { USA. }\end{array}$ \\
\hline
\end{tabular}

